\title{
Reason Analysis of Wheel Bag Crack in the Reliability Test of a Car and Structure Improvement Optimization
}

\author{
Han Yuan and Li Yuzhu \\ Wuxi Vocational Institute of Commerce, Wuxi, Jiangsu 214153, China
}

Keywords: Wheel bag cracking; Improvement of wheel bag structure; Reliability test

Abstract. The reasons for the problem of wheel bag fracture in specific models in the process of reliability test were analyzed in the paper, and according to the actual structure and process requirements, corrective measures were put forward, and the reliability was once again demonstrated and verified.

\section{Background}

A new development model was involved in the reliability test on the test field, and the accumulated mileage reached 10070 kilometers (3982 kilometers of strengthened road, see table 1 for the mileage of every road segment). The installation stent of the oil-water separator in the front cabin cracks, and the degree of cracking is serious. The specific cracking position is shown in figure 1 , and the cracking situation is shown in figure 2.

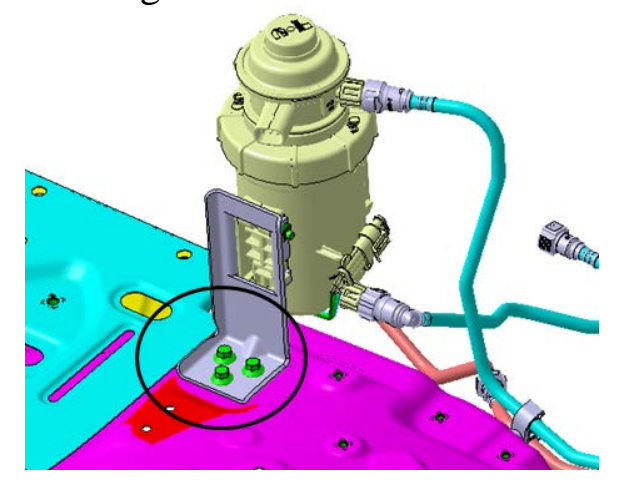

Fig.1 Cracking position of wheel bag (front cabin)

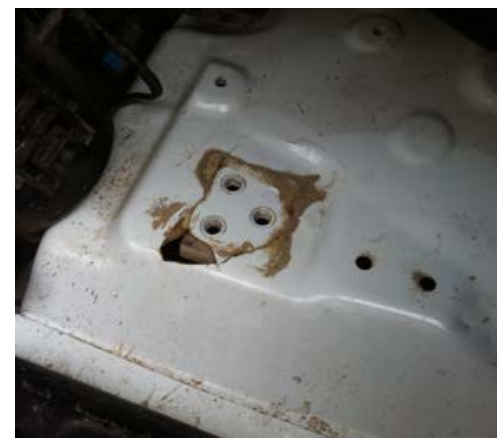

Fig.2 Cracking situation of wheel bag

Table 1 Mileage in case of cracking failure in the wheel bag.

\begin{tabular}{|c|c|c|c|c|}
\hline \multirow{4}{*}{$\begin{array}{c}\text { Mileage in } \\
\text { trouble }(\mathrm{km})\end{array}$} & Total mileage & \multicolumn{3}{|c|}{10070} \\
\cline { 2 - 5 } & Strengthened road & 3982 & Test running-in & 2502 \\
\cline { 2 - 5 } & High speed loop & 3006 & Mountain road & 530 \\
\cline { 2 - 5 } & General road & 0 & Other roads & 50 \\
\cline { 2 - 5 } & Mileage that day & 530 & Mileage that day & Mountain road \\
\hline
\end{tabular}




\section{Reason Analysis}

Structure and Material Analysis. The original data scheme is as follows:

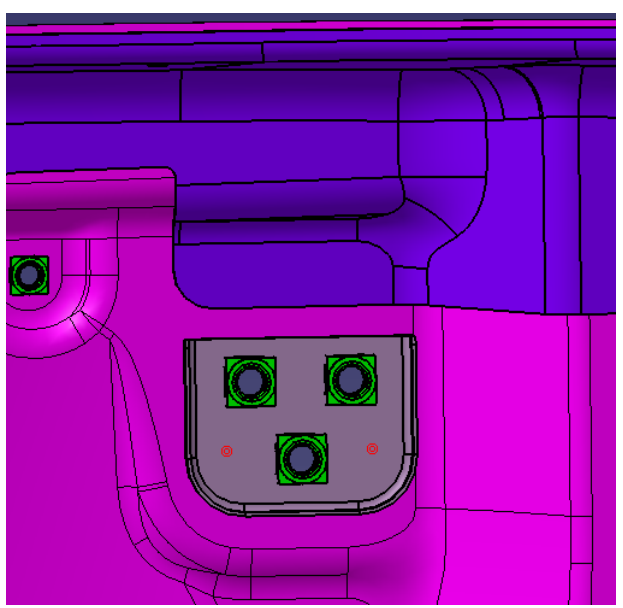

Fig.3 Wheel bag and wheel bag nut mounting plate

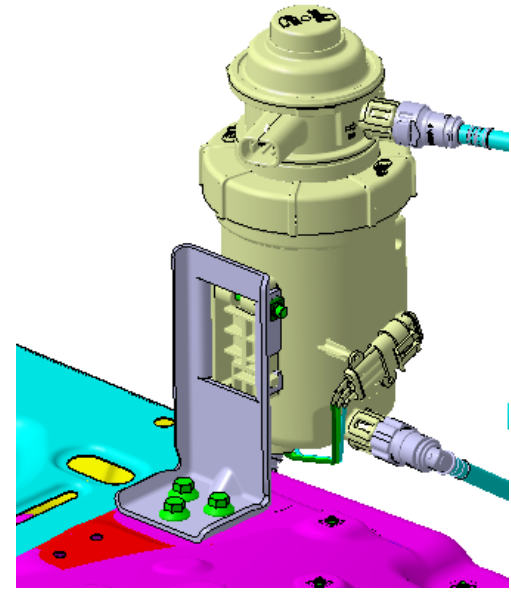

Fig.4 Mounting plate of oil-water separator stent

Table 2 Material properties

\begin{tabular}{|c|c|c|c|}
\hline Name & Material & $\begin{array}{c}\text { Thickness } \\
(\mathbf{m m})\end{array}$ & $\begin{array}{c}\text { Yield limit } \\
\mathbf{( M P a}\end{array}$ \\
\hline Wheel bag and wheel bag nut mounting plate & DC04 & 1.0 & 165 \\
\hline Mounting plate of oil-water separator stent & $510 \mathrm{~L}$ & 3.0 & 355 \\
\hline
\end{tabular}

Looking from the structure of the oil-water separator stent, a large cantilever is formed; regardless of the vehicle in the idling condition or in other bad conditions, large stress and stress deformation may be produced on the wheel bag and wheel bag nut mounting plate; with regard to the structure alone, this kind of structure has certain defects, and fails to meet the requirements of long-term vibration, with extremely high requirements for the materials. In addition, the installation surface of the oil-water separator stent is small, which will also have a bad effect on the wheel bag.

From a perspective of the oil-water separator stent and wheel bag material, the difference between the two is large, and whether the matching is reasonable is also a key point of this study.

Analysis of Finite Element Analysis Results. Firstly, the method of finite element analysis is used to preliminarily determine whether the current structure and materials are consistent with the problems in the reliability test.

It can be concluded from the analysis in figure 5: the finite element analysis results are basically consistent with the reliability test, and the maximum stress here in the $\mathrm{Y}$ and $\mathrm{Z}$ direction is beyond the yield limit (165MPa) of the material (DC04). 
To sum up, the cracking reasons for the wheel bag in the reliability test are subject 3 aspects as follows:

(1) The yield limit of the materials selected for the wheel bag and wheel bag nut mounting plate is too small, only 165MPa, which can not meet the requirement of use;

(2) The structure of the wheel bag nut mounting plate is unreasonable, which only plays the role of installation, but has not played any role in strengthening the wheel bag;

(3) The mounting surface of the oil-water separator stent is small, and the impact on the wheel bag is large and the phenomenon of stress concentration is generated in the process of vibration.

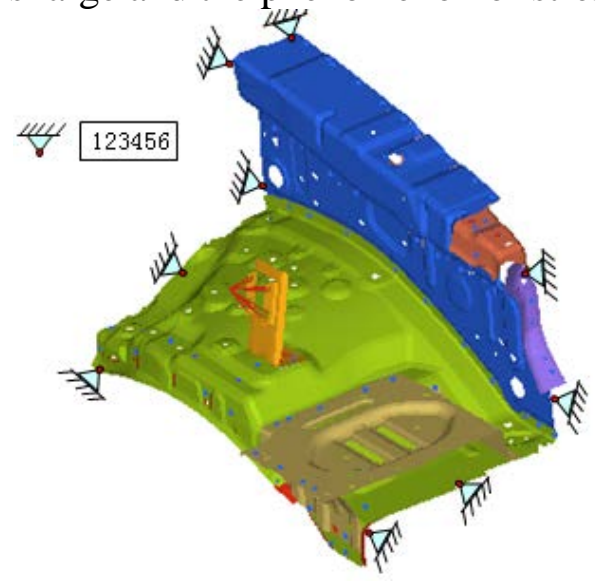

(a)Analysis model

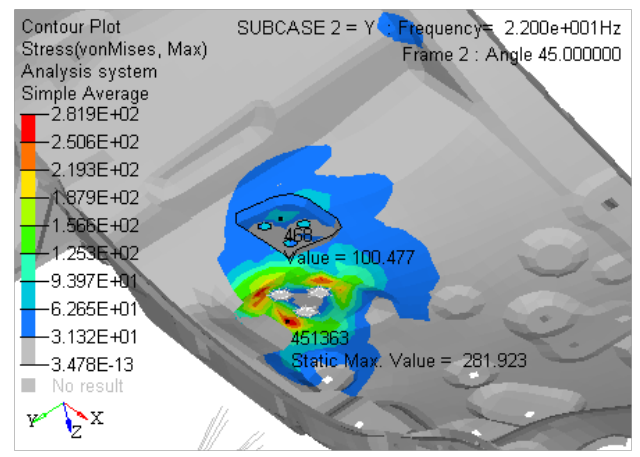

(c)Y-direction stress nephogram (maximum stress281.9MPa)

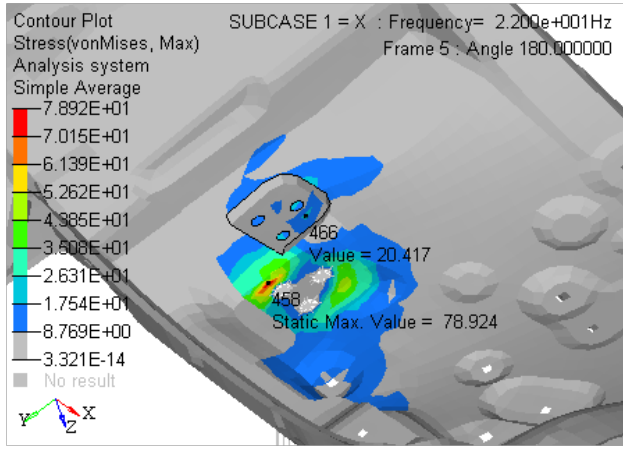

(b)X-direction stress nephogram (maximum stress78.9MPa)

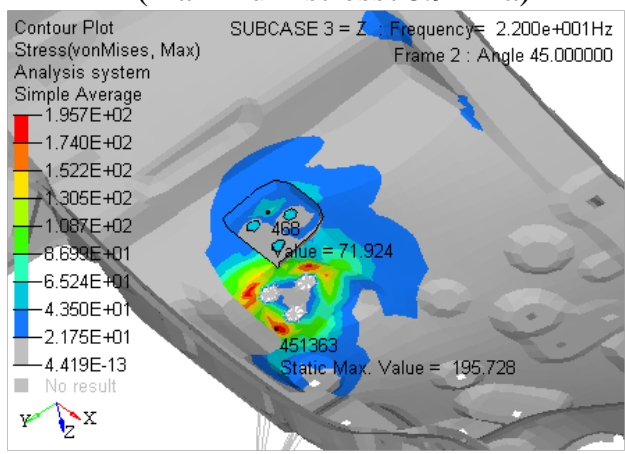

(d)Z-direction stress nephogram (maximum stress195.7MPa)

Fig. 5 The finite element analysis

\section{Improvement Design}

Because the wheel bag is large, and the ductility of stamping process and materials is highly required, the materials with large ductility were generally selected. Thus, this reason was fully considered in the process of the improved scheme put forward, and the material optimization was temporarily not proposed.

According to the above reasons, several improvement directions were proposed, and the method of finite element analysis was used to determine whether the three schemes can meet the requirements of reliability.

Scheme 1. It can be concluded from the stress nephogram of the original scheme that the strength of the wheel bag nut mounting plate is not enough, so the material thickness of the wheel bag nut mounting plate was increased, that is, the material thickness was increased from $1 \mathrm{~mm}$ to $1.8 \mathrm{~mm}$. The analysis results of scheme 1 are shown in figure 6 using the method of finite element analysis. 


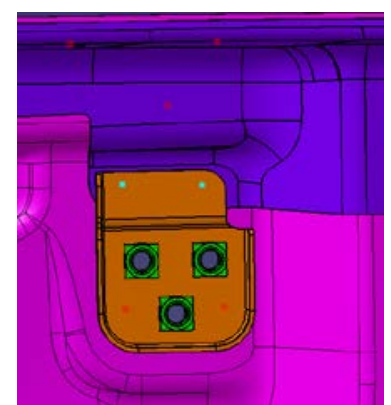

(a)Scheme 1 Thickening material and solder thickness

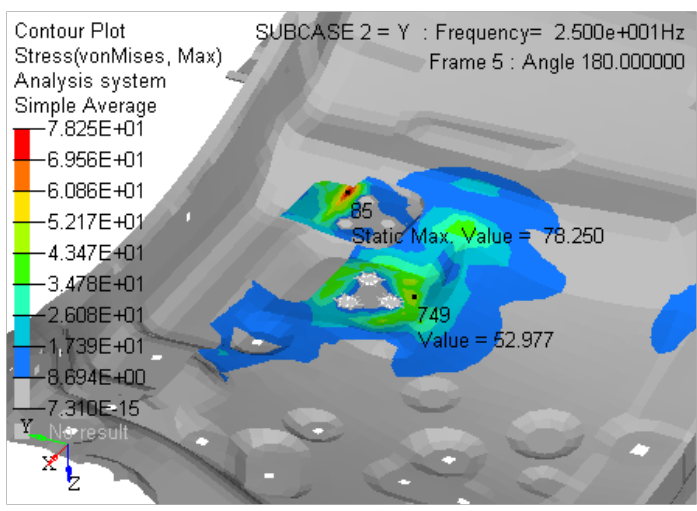

(c)Scheme 1 Y-direction stress nephogram (78.2MPa)

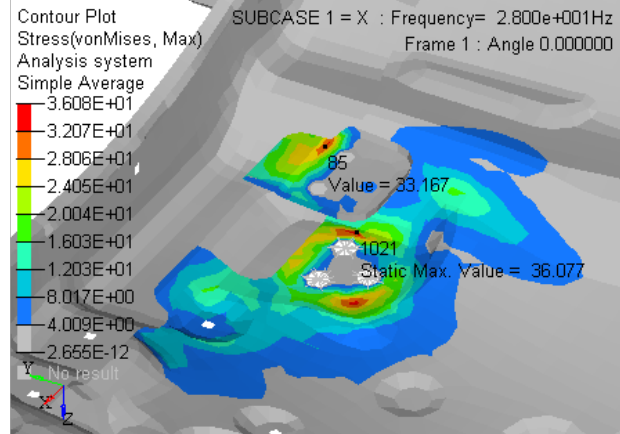

(b)Scheme $1 \mathrm{X}$-direction stress nephogram(36MPa)

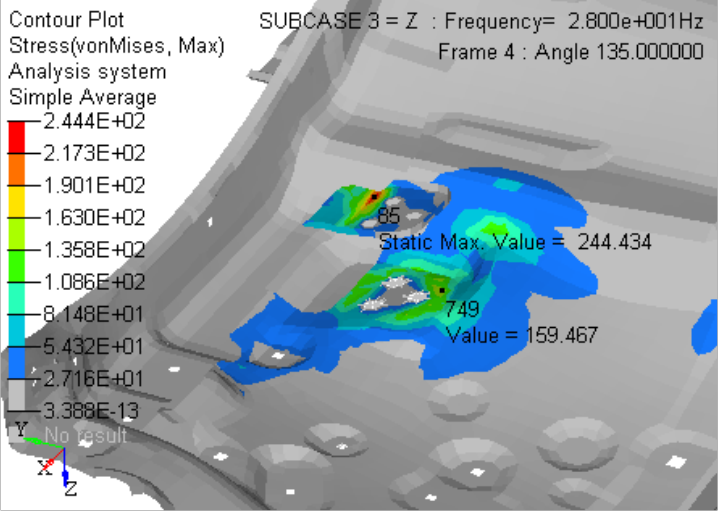

(d)Scheme 1 Z-direction stress nephogram

(244MPa)

Fig. 6 Scheme 1

Scheme 2. In the process of vibration, the stress concentration of the oil-water separator stent was generated on the wheel bag nut mounting plate. In order to disperse the concentrated stress, the mounting surface could be increased. The finite element analysis of scheme 2 was conducted, and the results are shown in figure 7.

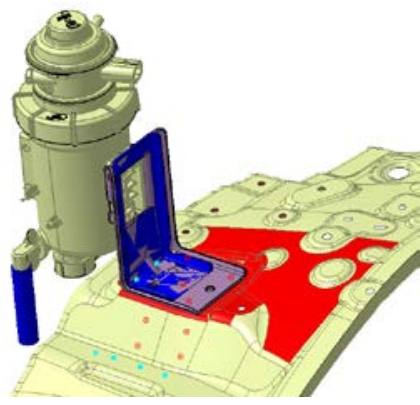

(a)Scheme 2 Increased mounting contact surface

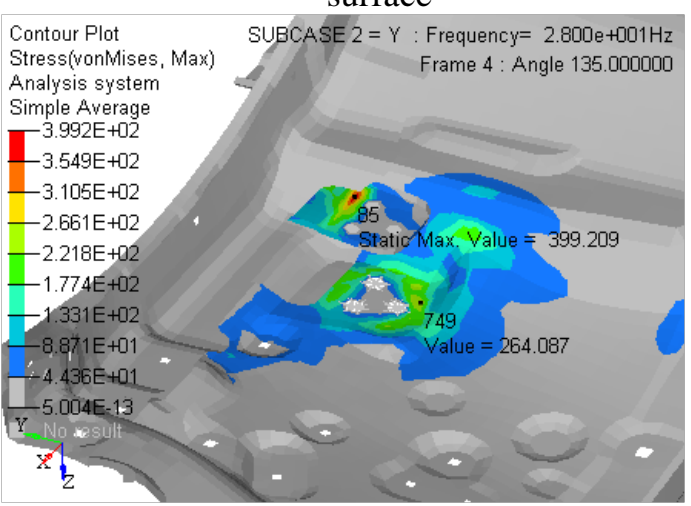

(c)Scheme 2 Y-direction stress nephogram (399.2MPa)

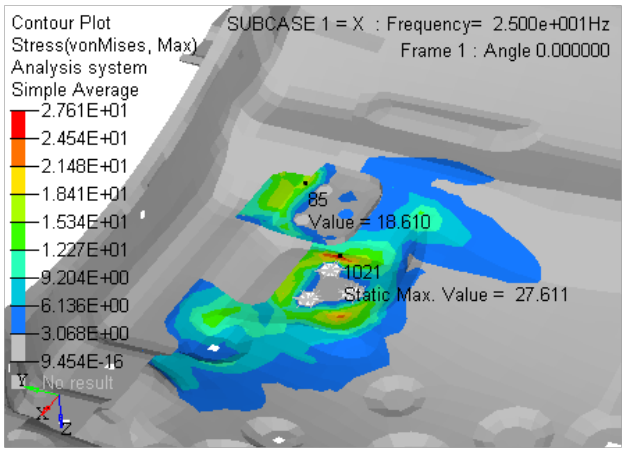

(b)Scheme 2 X-direction stress nephogram

(27.6MPa)

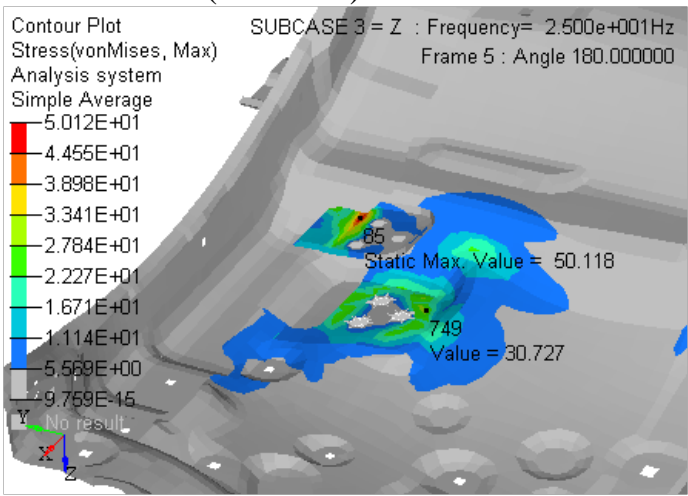

(d)Scheme 2 Z-direction stress nephogram (27.6MPa)

Fig.7 Scheme 2 
Scheme 3. Combined with scheme 2, the stress concentration was completely dispersed, and the whole wheel bag nut mounting plate was enlarged. The finite element analysis of scheme 3 was conducted, and the results are shown in figure 8.

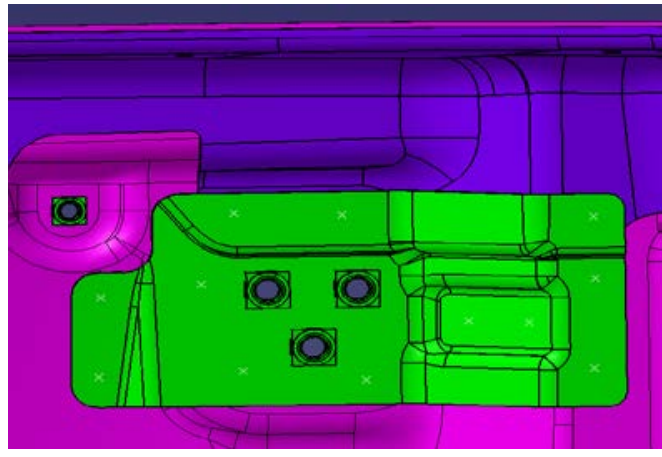

(a)Scheme 3 Overall increase in the wheel bag nut mounting plate

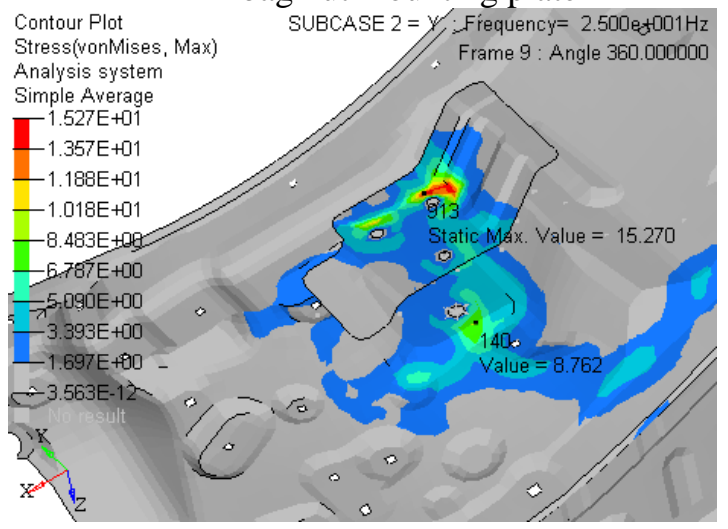

(c)Scheme 3 Y-direction stress nephogram $(15.2 \mathrm{MPa})$

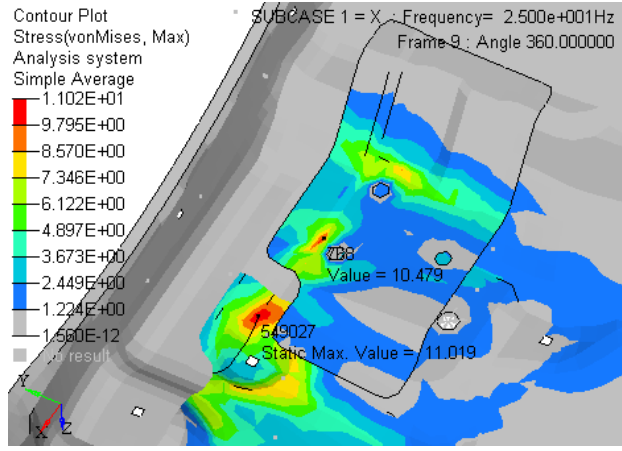

(b)Scheme $3 \mathrm{X}$-direction stress nephogram (11MPa)

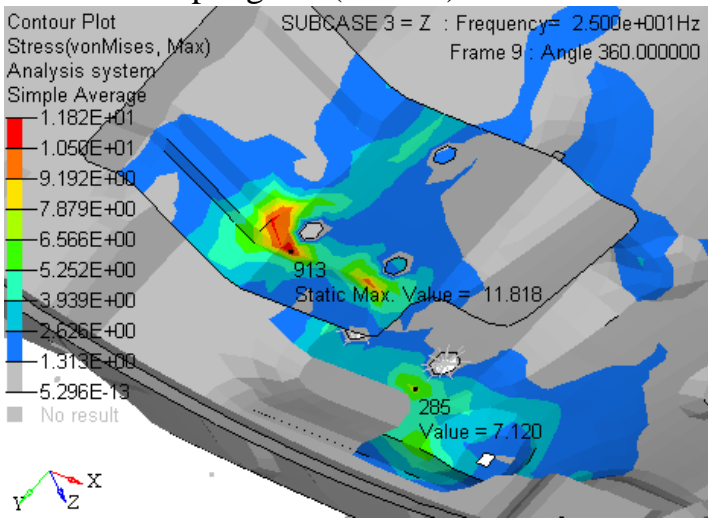

(d)Scheme 3 Z-direction stress nephogram

(11.8MPa)

Fig. 8 Scheme 3

It can be seen from the above stress nephograms that the nut mounting plates of the wheel bags in the schemes 1, 2 are beyond the yield limits of the selected materials, and the maximum stress of the wheel bag in the scheme 3 (the wheel bag nut mounting plate is overall increased) is only less than the largest yield limit of the selected material (165MPa), so samples are chosen from the scheme 3 for a new reliability test.

\section{Test verification}

The finite element analysis results can only be as a reference base, but can not serve as the delivery base of final products, because the scheme can be finalized only by complex reliability test in the complex conditions of the reliability test.

The picture below shows the physical photos of the oil-water separator and wheel bag nut mounting plate in the reliability test as well as the current reliability test mileage.

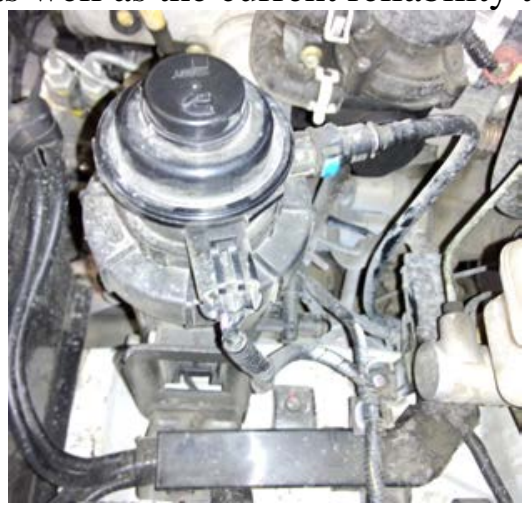

Fig.9 Front mounting position in the verification scheme 


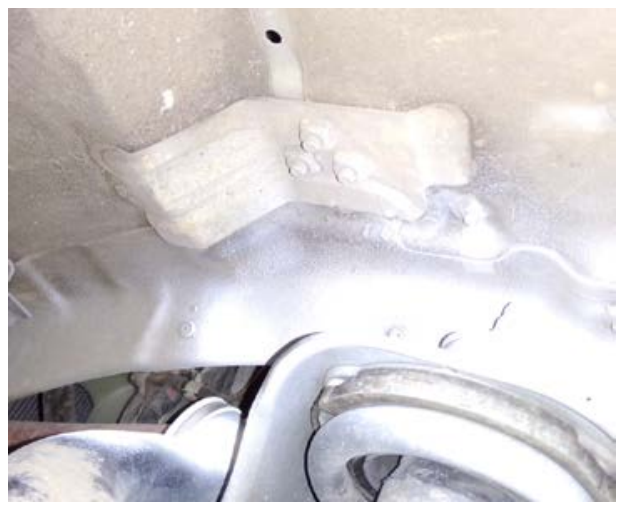

Fig.10 Rear mounting position in the verification scheme

Table 3 Mileage in case of fracturing failure in the wheel bag

\begin{tabular}{|c|c|c|c|c|}
\hline \multirow{4}{*}{$\begin{array}{c}\text { Mileage } \\
(\mathrm{km})\end{array}$} & Total mileage & \multicolumn{3}{|c|}{23819} \\
\cline { 2 - 5 } & Strengthened road & 11863 & Test running-in & 2501 \\
\cline { 2 - 5 } & High speed loop & 6000 & Mountain road & 3185 \\
\cline { 2 - 5 } & General road & 270 & Other roads & \\
\cline { 2 - 5 } & Mileage that day & 450 & Mileage that day & $\begin{array}{c}\text { Mountain } \\
\text { road }\end{array}$ \\
\hline
\end{tabular}

In order to test the improvement effect, the quality tracking was carried out every day in the following general road surface test, and no cracks here were found, indicating that the improved design is effective.

\section{Conclusion}

The raw data analysis results of wheel bag are consistent with problems in the reliability test of the real vehicle, showing that the crack phenomenon in the reliability test of the real vehicle results from unreasonable structure. It can also be seen from the stress nephogram of several schemes among the improvement schemes that the problem of stress concentration is radically solved by changing the structure, and ultimately, this is verified through the reliability test of the real vehicle. At the same time, it also gives designers a direction. Some fault problems in reliability tests should not be solved by directly changing the materials but can also be solved by other means (such as optimizing the structure).

\section{References}

[1] Wu Changqing, Liu Congcong. Analysis on frequency response of automotive wheel bag based on ANSYS [J] Shaanxi Auto, 2016(1):74-76.

[2] Wang Suifeng. Finite element analysis and optimization design of a small car frame structure [D]. Jiaotong University, 2008.

[3] Zheng Heyan, Lu Yaohui, et al. Finite element numerical simulation of structural side impact resistance of vehicle structure [J] Equipment Environment Engineering, 2017 (12):45-50.

[4] Wang Liang. Study on optimization design of vehicle support topology based on finite element technology [J]. Internal combustion engine and accessories, 2017 (17):40-41.

[5] Li Ping. Analysis on fracture behaviors of injection molded parts of automotive stents and technical study [D]. Nanchang University,2016.

[6] Li Guangpan, Guo Xuxun, Yang Bo, Wang Bin. Test method for endurance reliability of automobiles [J] Tianjin Automobile, 2008(10):34-37 